BULLETIN OF THE

AMERICAN MATHEMATICAL SOCIETY

Volume 77, Number 3, May 1971

\title{
BOUNDEDNESS AND OSCILLATION OF SOLUTIONS OF THE LIENARD EQUATION
}

\author{
BY JOHN R. GRAEF
}

Communicated by Wolfgang Wasow, November 18, 1970

1. Introduction. Consider the generalized Liénard differential equation

$$
x^{\prime \prime}+f(x) x^{\prime}+g(x)=e(t)
$$

of which

$$
x^{\prime \prime}+f(x) x^{\prime}+g(x)=0
$$

is a special case. We will assume throughout that $f$ and $g$ are continuous and $e$ is sectionally continuous. Many papers have been written giving sufficient conditions for boundedness or oscillation of solutions of (1) or (2), and surveys of results known prior to 1963 can be found in Sansone and Conti [3] and Reissig, Sansone, and Conti [2].

The study of equations (1) and (2) has fallen into two cases: first, the case where $f(x) \geqq 0$ for all $x$, and secondly, the case where $f(x)<0$ for $|x|$ small. The best results for the first case were given by Burton and Townsend [1] in the form of necessary and sufficient conditions for boundedness and oscillation of solutions of (1) in the case $x g(x)>0$ for $x \neq 0$. The results to be described here cover both of the cases mentioned above and generalize all results, including those in [1], which are known to the present time. The restrictions on the sign of $f(x)$ will be removed and instead placed on its integral. Furthermore, the conditions on the divergence of the integrals of $f$ and $g$ will be relaxed from those asked by previous authors.

\section{Uniform ultimate boundedness.}

DEFINITION. The solutions of (1) are uniformly ultimately bounded if there exists a constant $D>0$ such that for any solution $x(t)$ there is a time $T$ such that $|x(t)|<D$ and $\left|x^{\prime}(t)\right|<D$ for all $t>T$.

Define $F(x)=\int_{0}^{x} f(s) d s$ and $E\left(t, t_{0}\right)=\int_{t_{0}}^{t} e(s) d s$ and write (1) as the system

$$
\begin{aligned}
& x^{\prime}=y-F(x)+E\left(t, t_{0}\right), \\
& y^{\prime}=-g(x) .
\end{aligned}
$$

AMS 1969 subject classifications. Primary 3445; Secondary 3440, 3451.

Key words and phrases. Liénard equation, boundedness, forced oscillations. 
We make the following assumptions on system (3):

(4) For some $B>0,\left|E\left(t, t_{0}\right)\right| \leqq B$, for all $t \geqq t_{0}$.

Suppose there is a $k>0$ such that

(5) $F(x)-B>0$, for $x \geqq k$, and $F(x)+B<0$, for $x \leqq-k$.

(6) Either $F(x)-B \geqq A>0$, for $x \geqq k$, or $F(x)+B \leqq-A<0$, for $x \leqq-k$, for some fixed constant $A$.

(7) $x g(x)>0$, for $|x| \geqq k$.

THEOREM 1. Under conditions (4)-(7), all solutions of (1) are uniformly ultimately bounded if and only if $\int_{0}^{ \pm \infty}[f(x)+|g(x)|] d x= \pm \infty$.

The proof of this theorem consists of constructing a simple closed curve $C$ in the phase plane with the property that any solution $(x,(t), y(t))$ of (3) intersecting $C$ moves inside it, and all solutions which are outside of $C$ eventually intersect $C$.

To illustrate the technique of the construction while at the same time sparing the reader the details, let us consider the special case (2) of equation (1). Thus in conditions (5)-(7) let $B=0$.

Define $G(x)=\int_{0}^{x} g(s) d s$. Now we must consider the possible behaviors of $G(x)$ for $x>0$ and $x<0$, to obtain a total of four cases, one of which we will describe here. Suppose that $G(x) \nrightarrow \infty$ as $x \rightarrow+\infty$ and $G(x) \rightarrow \infty$ as $x \rightarrow-\infty$. Let $b=k+1, I=[-b, b]$, and define $L$ $=\max _{x \in I}|F(x)|$ and $K=\max _{x \in I}|g(x)|$. From (3) we obtain (recall that $e(t)=0)$

$$
\frac{d y}{d x}=\frac{-g(x)}{y-F(x)} .
$$

Choose $y_{0}<-L$ such that the curve $V_{0}=\left\{(x, y): V(x, y)=V\left(b, y_{0}\right)\right.$, $y<0\}$, where $V(x, y)=y^{2} / 2+G(x)$, is bounded away from the $x$-axis for $x \geqq b$. On the set $\left\{(x, y): x \in I,|y| \geqq\left|y_{0}\right|\right\},|d y / d x|<N$, for some $N>0$. Now choose the point $P_{1}=\left(b, y_{1}\right)$ such that $y_{1}<y_{0}-K / N$. We now define our curve $C$ (see Figure 1). $V_{1}=\left\{(x, y): V(x, y)=V\left(b, y_{1}\right)\right.$, $y<0, x \geqq b\}$.

$C_{1}$ is the straight line through $P_{1}$ with slope $N$ intersecting $x=-b$ at $P_{2}=\left(-b, y_{2}\right)$.

$C_{2}=\left\{(x, y): V(x, y)=V\left(-b, y_{2}\right), x \leqq-b\right\}$.

$C_{3}$ is the straight line through $P_{3}=\left(-b,\left|y_{2}\right|\right)$ with slope $N$ intersecting $x=b$ at $P_{4}$.

$C_{4}$ is the horizontal line through $P_{4}$ first intersecting $y=F(x)$ for $x>b$ at $P_{5}$.

$C_{5}$ is the vertical line through $P_{5}$ intersecting $V_{1}$ at $P_{6}$.

To show that any solution intersecting $C$ moves inside it, we need to show that the dot product of the tangent to the solution and the 


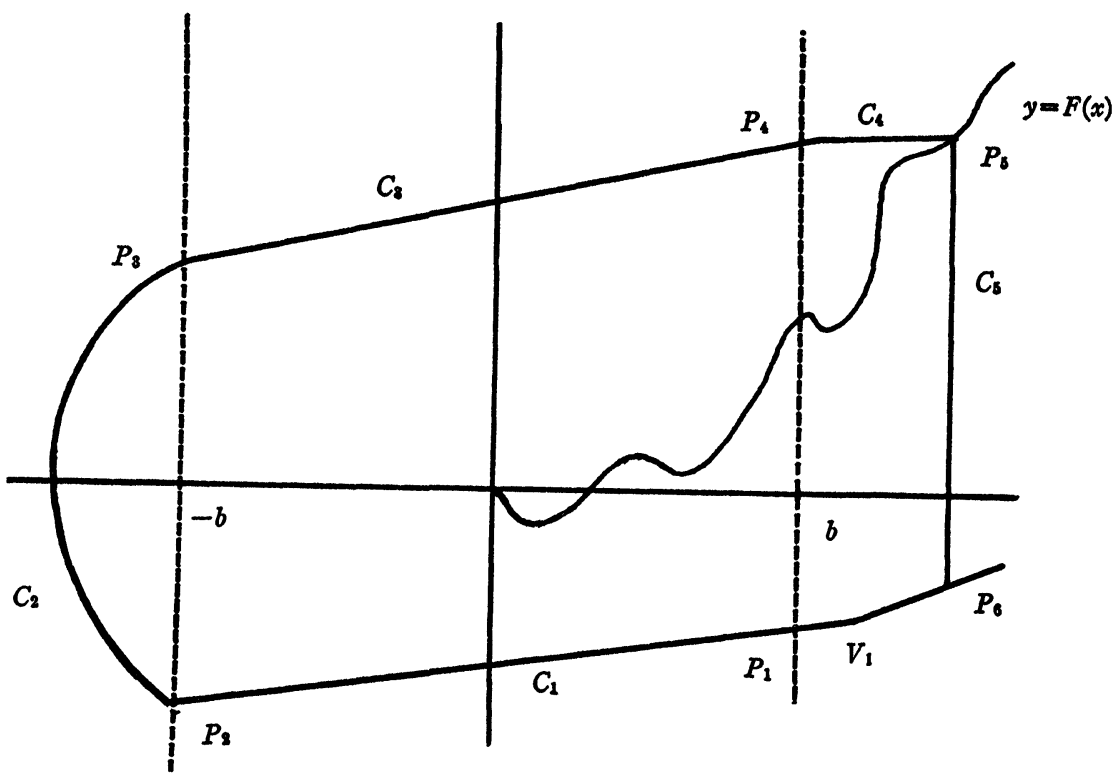

FIGURE 1

outward normal to the curve is negative. For example in the case of $V_{1}$,

$$
\begin{aligned}
V^{\prime} & =\operatorname{grad} V \cdot\left(x^{\prime}, y^{\prime}\right)=y y^{\prime}+g(x) x^{\prime} \\
& =-g(x) y+g(x)(y-F(x))=-g(x) F(x)<0
\end{aligned}
$$

for $x \geqq k$. Similarly for $C_{1}$, the outward normal is $(N,-1)$ so

$$
\begin{aligned}
(N,-1) \cdot\left(x^{\prime}, y^{\prime}\right) & =N(y-F(x))+g(x)<N(y+L)+K \\
& \leqq N\left(y_{1}+L\right)+K<N\left(y_{0}-K / N+L\right)+K<0
\end{aligned}
$$

by our choice of $y_{0}$. The details of the proof that all solutions must eventually intersect $C$ will be given in the forthcoming paper.

\section{Oscillation.}

Definition. A solution $x(t)$ of (1) is oscillatory if there is a sequence $\left\{t_{n}\right\}$ tending monotonically to $+\infty$ such that $x\left(t_{n}\right)=0$.

Suppose there exists $k>0$ such that

(8) $x F(x)>0$ for $|x| \geqq k$.

The following theorems are proved through a sequence of propositions some of which require constructions similar to the one described above.

THEOREM 2. If $x g(x)>0$ for $x \neq 0, f(0)<0$, and condition (8) holds, 
then all solutions of (2) oscillate if and only if $\int_{0}^{ \pm \infty}[f(x)+|g(x)|] d x$ $= \pm \infty$.

Theorem 3. Suppose $x g(x)>0$ for $x \neq 0$, conditions (4) and (8) hold, $e(t)$ is bounded, and $E\left(t, t_{0}\right)$ diverges. Then all solutions of (1) oscillate if and only if $\int_{0}^{ \pm \infty}[f(x)+|g(x)|] d x= \pm \infty$.

4. Remarks. Some corollaries which extend Theorem 1 have also been obtained. The propositions leading up to Theorems 2 and 3 actually yield stronger results in certain cases than are apparent from the statements of the theorems. An extensive bibliography surveying the literature will also be included in the forthcoming paper.

\section{REFERENCES}

1. T. A. Burton and C. G. Townsend, On the generalized Lienard equation with forcing term, J. Differential Equations 4 (1968), 620-633. MR 38 \#368.

2. R. Reissig, G. Sansone and R. Conti, Qualitative Theorie nichtlinearer Differentialgleichungen, Edizioni Cremonese, Rome, 1963. MR 28 \#1347.

3. G. Sansone and R. Conti, Non-linear differential equations, Macmillan, New York, 1964. MR 31 \#1417.

Mississippi State University, State College, Mississippi 39762 